\title{
CD44 mediates stem cell mobilization to damaged lung via its novel transcriptional targets, Cortactin and Survivin
}

\author{
Allal Ouhtit ${ }^{\bowtie}$, Rajesh Thouta1 ${ }^{1}$ Hatem Zayed ${ }^{2}$, Rajiv L. Gaur ${ }^{1}$, Augusta Fernando ${ }^{1}$, Mizanur Rahman ${ }^{1}$, \\ David A. Welsh ${ }^{3}$ \\ 1. Department of Biological \& Environmental Sciences, College of Arts \& Sciences, Qatar University, Doha, Qatar. \\ 2. Department of Biomedical Sciences, College of Health and Sciences, Qatar University, Doha, Qatar \\ 3. Section of Pulmonary/Critical Care Medicine and Allergy/Immunology, Louisiana State University Health Sciences Center, New Orleans, LA, 70112, USA. \\ $\square$ Corresponding author: Allal Ouhtit, PhD, MPh, Professor, Department of Biological and Environmental Sciences, College of Arts and Sciences, Qatar \\ University, Doha, Qatar. Phone: 00974-4403-7572; Fax: 00974-4403-453; E-mail: aouhtit@qu.edu.qa
}

(C) The author(s). This is an open access article distributed under the terms of the Creative Commons Attribution License (https://creativecommons.org/licenses/by/4.0/). See http://ivyspring.com/terms for full terms and conditions.

Received: 2019.01.14; Accepted: 2019.05.17; Published: 2020.01.01

\begin{abstract}
Beyond their role in bone and lung homeostasis, mesenchymal stem cells (MSCs) are becoming popular in cell therapy. Various insults may disrupt the repair mechanisms involving MSCs. One such insult is smoking, which is a major risk factor for osteoporosis and respiratory diseases. Upon cigarette smoke-induced damage, a series of reparatory mechanisms ensue; one such mechanism involves Glycosaminoglycans (GAG). One of these GAGs, namely hyaluronic acid (HA), serves as a potential therapeutic target in lung injury. However, much of its mechanisms of action through its major receptor CD44 remains unexplored. Our previous studies have identified and functionally validated that both cortactin (CTTN: marker of motility) and Survivin (BIRC5: required for cell survival) act as novel HA/CD44-downstream transcriptional targets underpinning cell motility. Here, human MSCs were treated with "Water-pipe" smoke to investigate the effects of cigarette smoke condensate (CSC) on these HA-CD44 novel signaling pathways. Our results show that CSC decreased the expression of both CD44 and its downstream targets CTTN and BIRC5 in MSCs, and that HA reversed these effects. Interestingly, CSC inhibited migration and invasion of MSCs upon CD44-targeted RNAi treatment. This shows the importance of CD44-HA/CTTN and CD44-HA/BIRC5 signaling pathways in MSC motility, and further suggests that these signaling pathways may provide a novel mechanism implicated in migration of MSCs during repair of lung tissue injury. These findings suggest that one should use caution before utilizing MSC from donors with history of smoking, and further pave the way towards the development of targeted therapeutic approaches against CD44-associated diseases.
\end{abstract}

Key words: mesenchymal stem cells, cigarette smoke, CD44, Cortactin, Survivin

\section{Introduction}

Cigarette smoking is a common risk factor for numerous systemic conditions, including respiratory diseases and osteoporosis. Cigarette smoke can cause DNA damage, free radical production, inflammation and lung cell injury, all of which negatively affect homeostasis of the lung [1-4]. Furthermore, it disrupts various cellular mechanisms implicated in the repair of the damage [1-4].

Bone marrow mesenchymal stromal cells (MSCs) participate in the maintenance and repair of bone as well as acts as a reservoir of progenitor cells for various solid organs. This "reservoir" capacity allows it to contribute to the regeneration of mesenchymal tissues in the event of injury [1-4]. In the lung, MSCs play a pivotal role in the regeneration of almost all cell types, thereby boosting repair mechanisms [1-4]. Beyond their role in bone homeostasis, ex vivo expanded MSCs have also attracted considerable attention for their potential in cell therapy of a wide variety of pathological conditions [2-7]. However, this potential of MSCs is detrimentally affected by cigarette thus reducing their therapeutic potency $[8,9]$.

Glycosaminoglycans (GAGs) can protect against cigarette smoke-induced damage by potentiating 
repair process of smoke-mediated DNA damage. Indeed, when normal proteoglycan synthesis is disrupted using specific proteoglycan inhibitors, emphysematous changes and parenchymal destruction were observed [1-4]. Furthermore, senescence and emphysema have been associated with a decrease in the GAG species of the lung [1-4]. In particular, hyaluronan or HA has been reported to play a critical role in the repair process of lung tissue in emphysema. Reduction in HA content is also correlated with increased degradation of elastin by elastases [1-4]. This is likely due to the fact that HA, along with other GAGs, can form a protective barrier that prevents access of elastases to elastin [1-4]. In animal studies, intratracheal or aerosolic HA supplementation protected elastin fibers from degradation, a role that was compromised by hyaluronidase and lysozyme [1-4].

Binding HA to CD44 is involved in a multitude of functions, including cell adhesion, motility, angiogenesis and inflammation [10,11]. Indeed, CD44 is one of the most abundant receptors on MSC and plays a major role in cell migration in response to chemotactic stimuli. The signaling pathways involved during HA-CD44 interaction have been mostly investigated in cancer [12]. Several studies have established a positive correlation between cell invasiveness and increased expression of HA, HAS2 (HA synthase 2), HYAL2 (hyaluronidase 2), and CD44 $[13,14]$. However, the molecular pathways implicated in HA-CD44-induced migration of MSCs stimulated by nicotine, a key component of cigarette-smoke, are nascent.

We have previously identified and validated Cortactin (CTTN) [15], Survivin (BIRC5) [16], and TGF- $\beta$ [17] as novel downstream transcriptional target genes in the HA/CD44 signaling pathway. Nicotine decreased CD44 in rodent bone marrow stromal cells [18]. Here, we examined the role of CD44-HA interaction in migration of MSCs exposed to cigarette smoke. We provide exciting evidence implicating CD44-HA/CTTN and CD44-HA/BIRC5 in MSC cell motility. Taken together, our results suggest that these signaling pathways may provide a novel target in modulating migration of MSCs during repair of lung tissue injury.

\section{Results}

\section{Establishment of the optimal dose of CSC affecting CD44}

Prior to examining the effect of CSC in MSCs, AlamarBlue ${ }^{\circledR}$ proliferation assay was utilized to determine the optimal dose of CSC required for all the experiments (Figure 1). MSC cells were seeded at $2 \times 10^{4}$ cells on Costar black tissue culture plates and were allowed to attach overnight. The cells were treated with medium containing various doses of CSC. AlamarBlue ${ }^{\circledR}$ was added to all the wells. Cells were observed at appropriated time points for morphological changes and fluorometric readings were taken at excitation of $540 \mathrm{~nm}$ and emission $610 \mathrm{~nm}$.

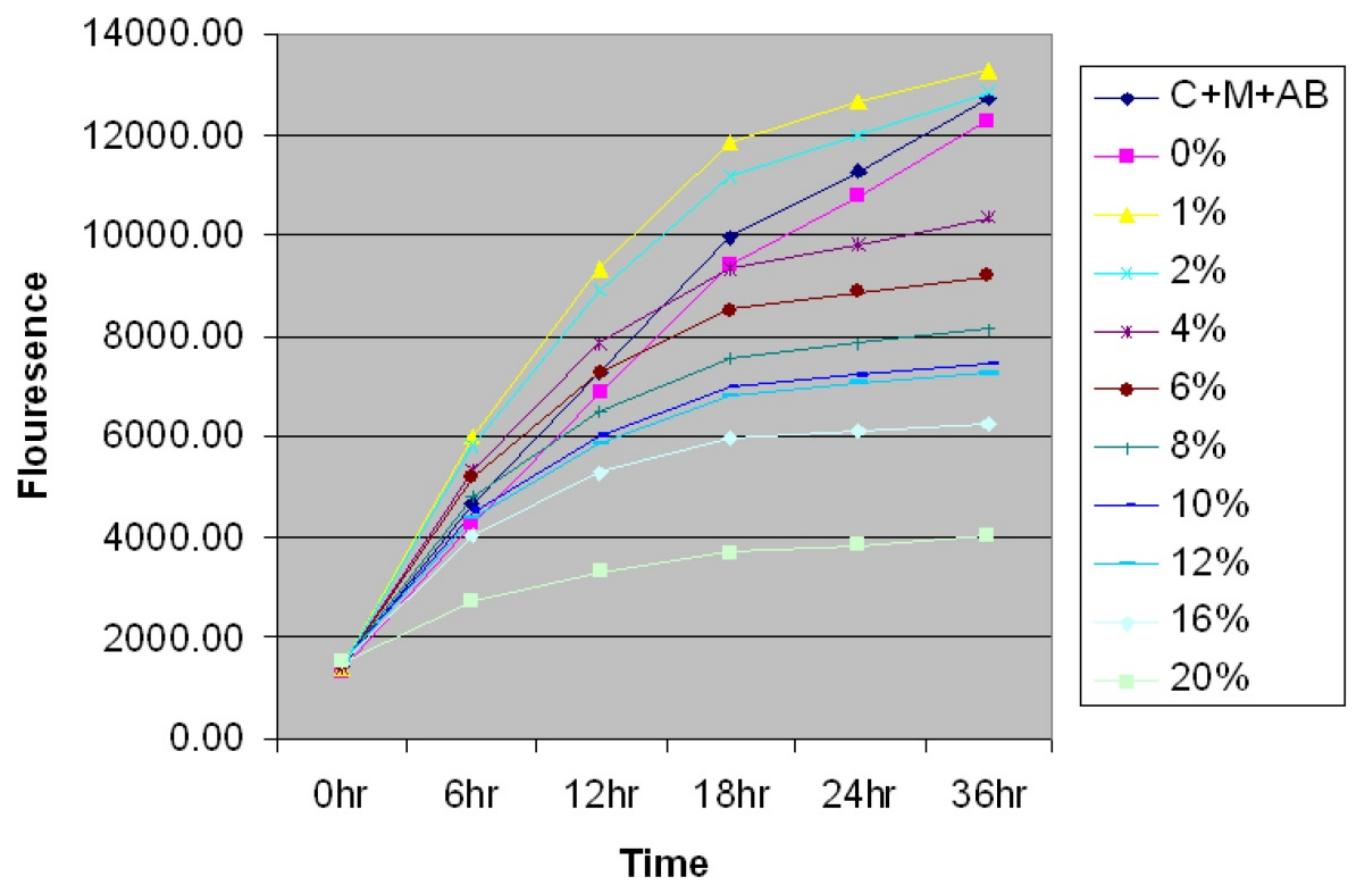

Figure 1. Time- and dose-dependent effect of CSC on the proliferation of MSC. Time and dose-dependent effect of CSC on the proliferation of MSCs. Based on the growth curves obtained, $6 \%$ CSC ( $\sim 80 \%)$ was selected as an optimal dose for further experiments. 
A

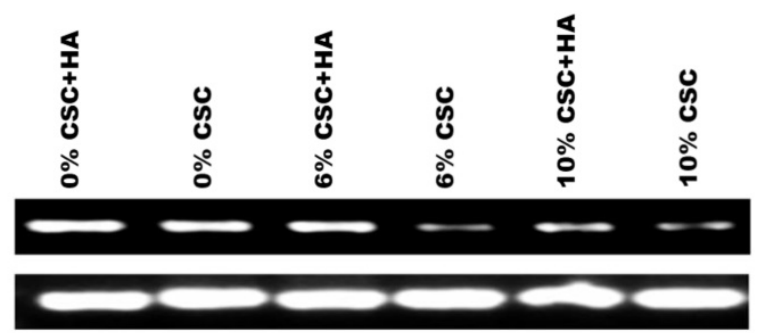

B

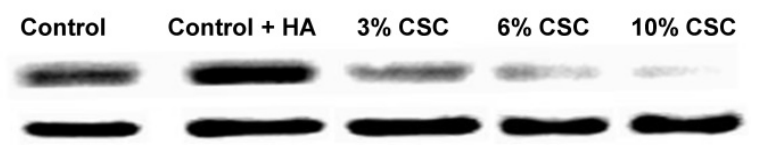

Figure 2. CSC decreases both CD44 RNA and protein Expression levels in MSC. (A) CSC decreases CD44 RNA expression levels in MSC. (B) CSC decreases CD44 protein expression levels in MSC. Hyaluronic Acid ameliorates CSC effect on CD44 Expression in MSC.

The results showed a time and dose-dependent effect of CSC on the proliferation of MSCs. Interestingly cell proliferation decreased in due course of time rather than an abrupt change indicating an induction of cell death by specific mechanism rather than a toxic effect of CSC. Based on the growth curves obtained, $6 \%$ CSC ( $80 \%)$ was selected as an optimal dose to evaluate the actions of CSC on MSCs.

\section{CSC decreases both CD44 RNA and protein Expression levels in MSC}

Hylorunan $(100 \mu \mathrm{g} / \mathrm{ml})$, a known ligand of CD44 showed high expression of CD44 expression was used as a control (Figures 2A and B). The RT-PCR results showed a marked decrease in the expression of CD44 mRNA with increasing concentrations of CSC in the media (Figure 2 A). Similarly, the effect of CSC on CD44 protein expression was performed by western blot analysis showing a decrease of CD44 protein.

\section{HA ameliorates the CSC effect on CD44 expression in MSC}

After establishing the effect of CSC in decreasing the expression levels of CD44 both at RNA and protein levels, we tested the hypothesis whether HA could reverse this effect (Figure 3 ). When MSCs were treated with increasing concentrations of CSC, dose-dependent decrease in CD44 protein expression was observed (Fig. 3A). Interestingly however, treatment with HA completely abolished CSC's effect on CD44 protein expression (Fig. 3A). These results were further confirmed by immunocytochemistry, showing similar expression patterns of CD44 in both control and CSC-treated MSCs in the absence or presence of HA (Fig. 3B).

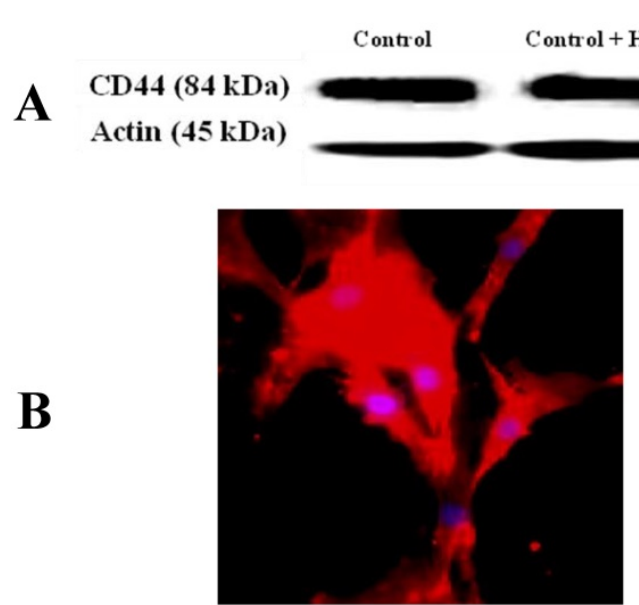

Control

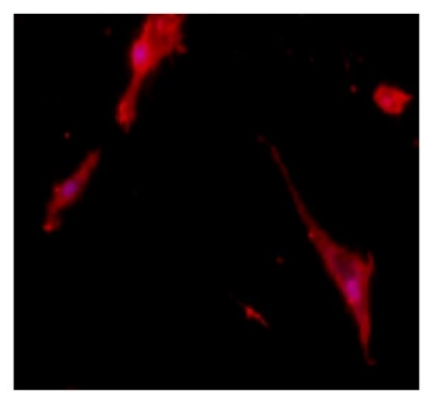

CSC

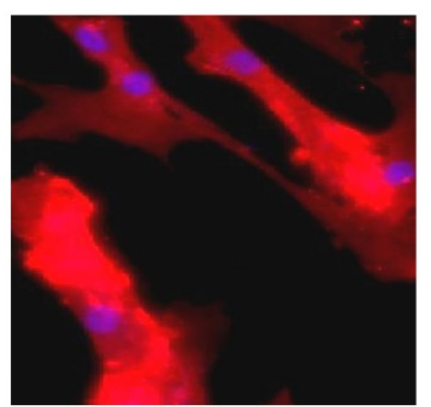

Control + HA

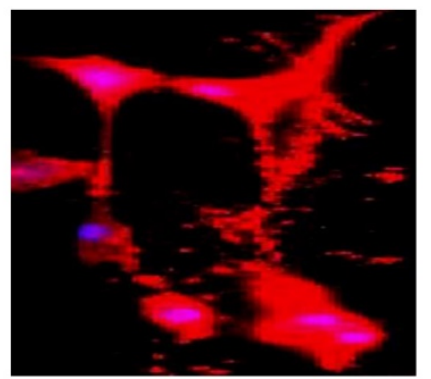

$\mathrm{CSC}+\mathrm{HA}$

Figure 3. Hyaluronan (HA) ameliorates the CSC effect on CD44 expression in MSC. (A) While CSC decreased CD44 protein expression, treatment with HA reversed CSC's effect on CD44 protein expression. Shown are the cropped blot images representing indicated proteins, and all the gels from three separate experiments have been run under the same experimental conditions. (C) Immunocytochemical images showing hyaluronic acid ameliorating CSC effect on CD44 Expression in MSC. 
A

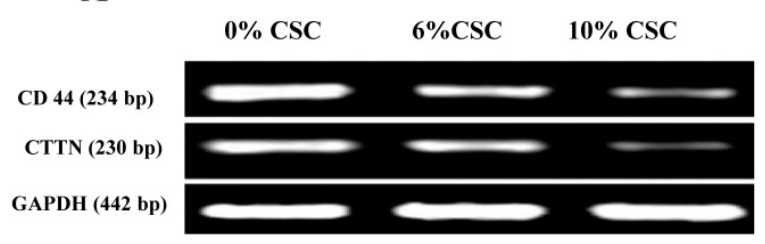

B

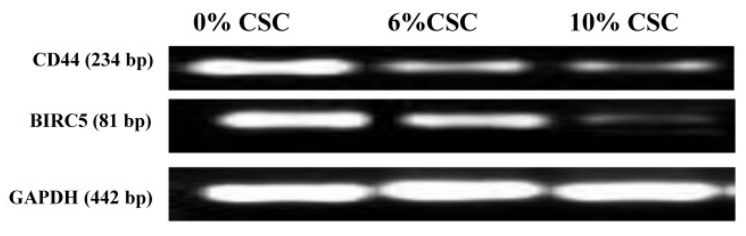

Figure 4. CSC inhibits both Cortactin and Survivin RNA expression levels parallel to CD44 expression. (A) CSC-reduced CD44 RNA levels paralleled CTTN RNA expression levels. (B) CSC-reduced CD44 RNA levels paralleled BIRC5 RNA levels. Shown are the cropped blot images representing indicated proteins, and all the gels from three separate experiments have been run under the same experimental conditions.

\section{CSC inhibits both Cortactin and Survivin Expression levels parallel to CD44}

We have recently discovered that CD44 can impair cancer cell migration and invasion via its two novel transcriptional downstream targets, CTTN [15], Survivin (BIRC5) [16]. To test whether CSC-decreased CD44 expression also decreases its transcriptional targets, CTTN and BIRC5, we examined their respective RNA (Figure 4) and protein (Figure 5) expression levels, using RT-PCR and western blot analyses, respectively.

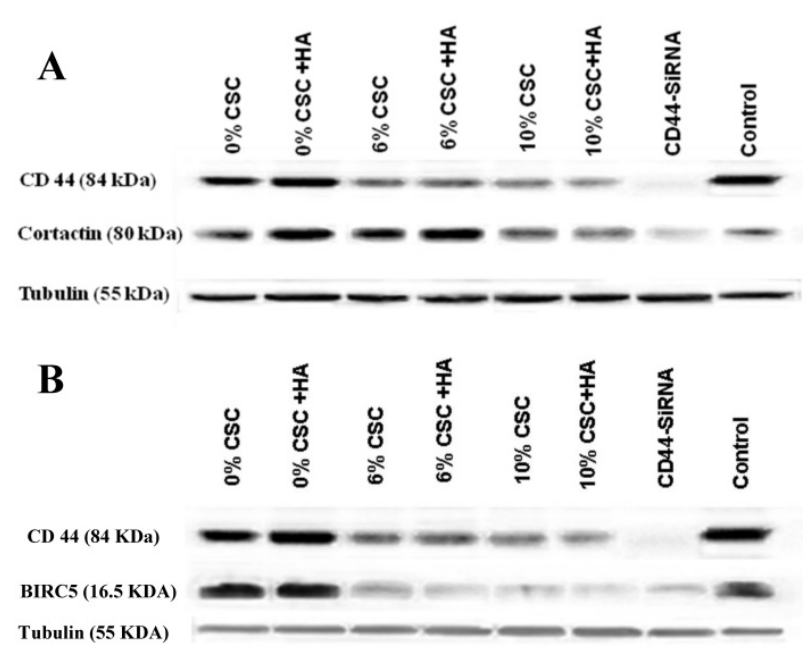

Figure 5. CSC inhibits both Cortactin and Survivin protein expression levels parallel to CD44 protein expression. (A) CSC-reduced CD44 protein expression levels paralleled CTTN protein expression levels. (B) CSC-reduced CD44 protein expression levels paralleled BIRC5 protein expression levels. Shown are the cropped blot images representing indicated proteins, and all the gels from three separate experiments have been run under the same experimental conditions.

In fact, when CD44 expression levels were reduced both at RNA and protein levels in response to CSC treatment of MSCs, both CTTN and BIRC5 expression levels were reduced (Figures 4 and 5). More interestingly, inhibition of CD44 using specific CD44 siRNA primers showed the same patterns of inhibition of both CD44 transcriptional targets, CTTN and BIRC5 (Figures 4 and 5). Taking together, CSC-inhibited CD44 expression appears to directly affect its CTTN and BIRC5 downstream target signaling pathways in response to CSC treatment of MSCs.

\section{CSC impairs migration and invasion properties of MSC, mediated through CD44 signaling}

To test whether CSC treatment of MSCs affecting CD44 signaling also affects its associated function in MSC migration and invasion, we initially optimized CD44 inhibition, using different doses of siRNA as shown in Figure 6A. The decrease of CD44 expression was dose-dependent of siRNA concentration.

Similarly to the experiments performed above, MSC Cells were treated with CSC (6\%) and (10\%) doses and with media containing siRNA specific to CD44. The Boyden chamber assay was carried out to measure both migration (absence of Matrigel) as well as MSC invasion (presence of Matrigel). In all cases, the results showed a marked decrease in migrated (Fig. 6B) and invaded (Fig. 6C). These results put together suggest that mobilization of MSC cells to damaged lung might be mediated by CD44 signaling pathways.

\section{Discussion}

In this study, we examined the effect of cigarette smoke on CD44-mediated MSC migration and invasion. Our results showed a marked decrease in migrating cells after CSC exposure; this is consistent with a previous study demonstrating that CSC attenuated fibronectin and platelet-derived growth factor stimulated chemotaxis, accompanied by a detrimental effect on the regenerative capacity of MSCs [19].

Cigarette smoke may lead to cell death, inhibit MSC growth, and reduce their ability to migrate and engraft while increasing their senescence [19]. It has also been reported that smoke interferes with the function and the normal processes in lung fibroblasts and bone marrow stromal cells [20,21]. Our results showed a consistent temporal and quantitative relationship between CSC and MSC, especially at lower concentrations. Concentration-dependent decrease in proliferation of MSCs was observed over a period of 36 hours, suggesting that growth inhibition is unlikely induced by direct toxic effect. Interestingly, low doses of CSC stimulated proliferation, while higher concentrations decreased MSC proliferation. 

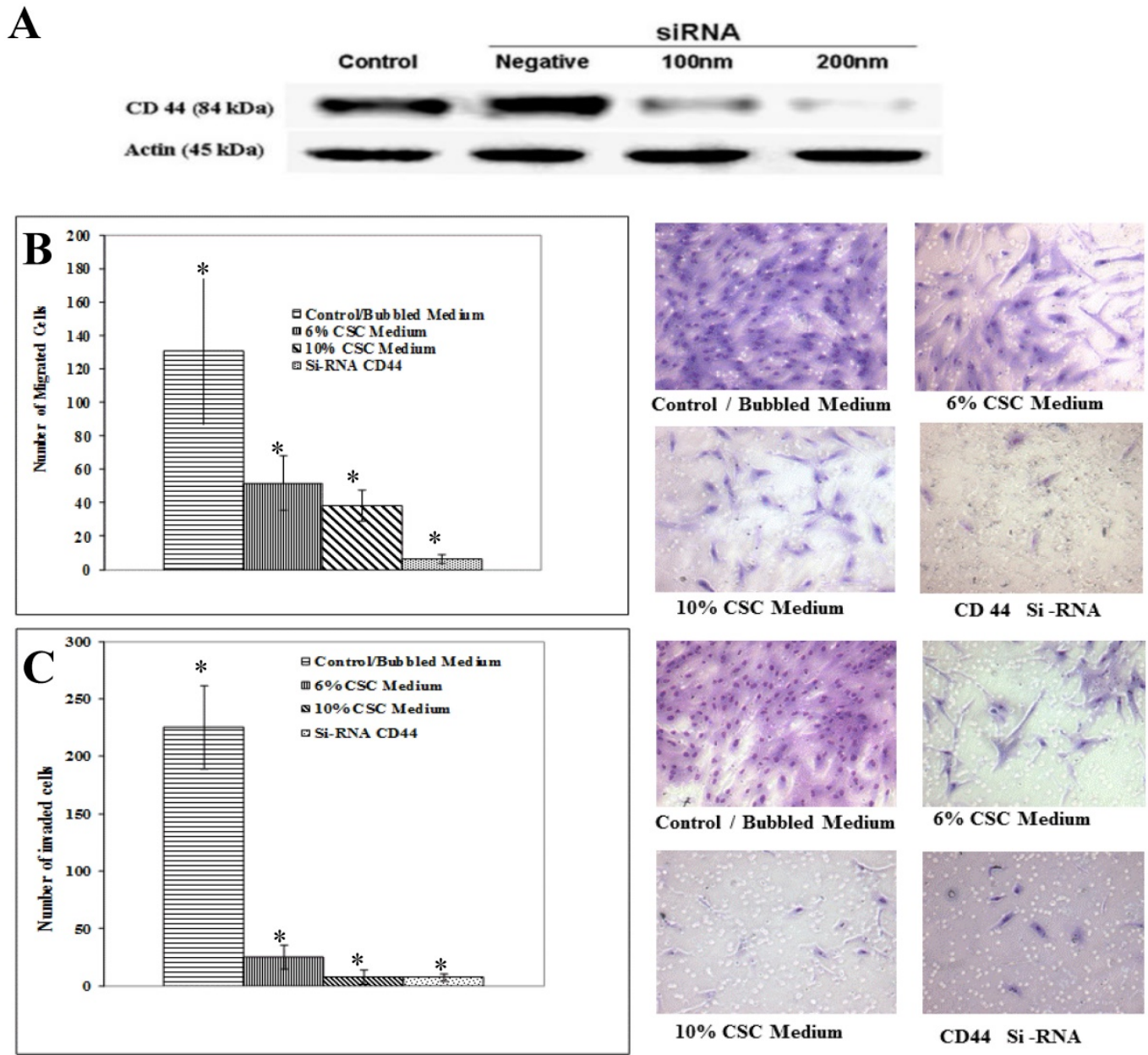

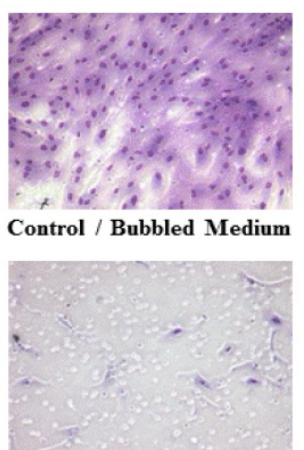

$10 \%$ CSC Medium
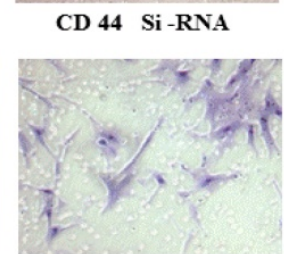

6\% CSC Medium

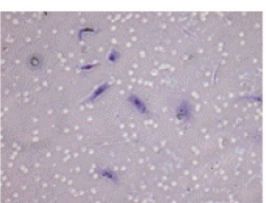

CD44 Si-RNA

Figure 6. CSC impairs migration and invasion ability of MSC mediated through CD44. (A) Gradual suppression of CD44 expression using CD44 SiRNA. CD44 inhibition was optimized by different doses of siRNA. Shown are the cropped blot images representing indicated proteins, and all the gels from three separate experiments have been run under the same experimental conditions. (B) Representative images of Boyden chamber membranes represent the number of captured cells during migration, illustrating the difference in migrated cell numbers. (C) Graphical representation of invading cells was demonstrated by invasion assay. Note a marked decrease in MSC cell migration and invasion capabilities after inhibiting CD44 expression using siRNA. Control cells were cultured in the same conditions. Representative images of Boyden chamber membranes represent the number of captured cells during the invasion, illustrating the difference in invaded cell numbers. The difference is considered statistically significant (student's t-test $* \mathrm{P}<0.001$ ).

$\mathrm{CD} 44$ is a transmembrane glycoprotein abundantly expressed on the surface of MSCs, and commonly used as a characteristic marker for MSC. CD44 is essential in the hematopoiesis process, tumor metastasis, lymphocyte activation, and MSC homing $[10,11,22]$. It also serves as a link between cells and the extracellular matrix, regulating cell adhesion and migration [10,11,22]. While HA is its major ligand, CD44 can also be activated by other ligands such as osteopontin, collagens and fibronectin [11,23-27].

Nicotine is one element of cigarette smoke that inhibits CD44 expression in a group of bone marrow stromal cells and in bone marrow derived-endothelial cell line (STR-12) $[18,28,29]$. In our study, we found that in response to CSC, a concentration-dependent decrease in CD44 expression was observed at both mRNA and protein levels. Paradoxically, several studies have reported an increase in soluble CD44 in smokers, thus suggesting increased production and/or increased shedding [30-32]. However, our data suggests a transcriptional level inhibitory effect, which is not consistent with shedding as the mechanism for reduced CD44 expression in our experimental setting.

There is a significant evidence supporting the role of CD44 in migration and homing of MSC; Zhu et al., 2006 have demonstrated that CD44 facilitated MSC adhesion and migration in murine CD44\%- bone marrow stromal cells [33]. Sackstein et al., demonstrated that induction of an alternative CD44 glyco-form on MSCs facilitated their homing [34], suggesting the involvement of CD44 in MSC motility. Our results support this finding, as RNAi inhibition of CD44 clearly suppressed migration and invasion of MSC.

HA is the major ligand for CD44 In emphysema, HA forms a complex with other GAGs to provide a protective shield that blocks access of CSC to molecules such as elastin, [35]. HA also targets CD44 and inhibits FcepsilonR1 signaling that involves ROS 
and MAPK [18,36]. Our results showed that CSC-induced reduction in CD44 expression was abolished when cells were treated with HA (Fig. 2). This confirms the protective effect that HA imparts against CSC-induced cell damage. Interestingly, smoking increases mucin production and mucus hypersecretion in a ROS-dependent manner [34]. This aggravated mucus secretion in airway epithelia appears to be directly related to depolymerization of hyalouronan [34]. Depolymerization of HA is pathologically an important process, since functions of HA are size-dependent, and low-molecular-weight HA is considered a "danger signal" [34].

We have previously demonstrated using Microarray analysis that CD44 promotes breast cancer cell invasions and metastasis to the liver [37], and identified CTTN [15] and BIRC5 [37] as downstream target genes underpinning CD44-promoted cell survival, migration and invasion. While CTTN is a marker of motility, due to its close relation to the actin cytoskeleton, BIRC5 is an elusive protein required for the survival of the cells, and these two proteins play a key role in lung injury $[16,38]$. The results of the present study revealed that CSC inhibited the expression of CD44, which in turn downregulated the expression of both its downstream transcriptional targets, CTTN and BIRC5. This parallel decrease of CTTN and BIRC5 was confirmed by RT-PCR, and further confirmed by RNAi inhibition of CD44 leading to significant reduction in CTTN and BIRC5 expression. Evidence supports a role of CD44 for homing of hematopoietic precursor cells and T-lymphocytes [39]. A previous study by Sackstein et. al., 2008, demonstrated induction of an alternative CD44 glycoform on MSC, which facilitated their homing to the bone, suggesting the importance of CD44 in MSC motility [34]. Our results support this finding, as inhibition of CD44 with specific iRNA strongly suppressed migration and invasion of MSC cells (Fig. 4). Inhibition of CD44 with RNAi strongly suppressed migration and invasion of the MSC cells. This indicates the importance of CD44-downstream signaling in MSC motility [34]. These data providing a clear evidence that CD44 signaling are vital for migration and invasion of MSC are supported by recent studies, emphasizing the regenerative potential of MSCs in the development of future cell-based clinical trials and therapies.

In summary, this study demonstrates the potential involvement of CD44 in the impairment of MSC migration and invasion in cigarette smokers (or in response to cigarette smoke). Furthermore, our findings suggest that this mechanism is mediated through a dual transcriptional regulation of CTTN and BIRC5 by CD44. These findings provide insights into potential novel mechanisms by which smoking may contribute to the development of osteoporosis, lung injury and emphysema, and further suggest that a caution must be taken before using smokers' MSC as source for cell therapy. In addition to a better understanding of the mechanisms by which CD44 mediates cigarette smoke-suppressed MSC cell motility, this investigation identified CD44/CTTN and CD44/BIRC5 signaling pathways as potential targets to pave the way towards the design of therapeutic strategies against cancer, emphysema and other CD44-associated diseases [40].

\section{Materials and Methods}

\section{Cell Culture}

Human bone marrow derived human MSC (MSC) were obtained from the Tulane Center of Gene Therapy (New Orleans, LA) and cultured in $\alpha$-MEM medium containing 16.5\% FBS (v/v, Atlanta Biologicals, Miami, FL), $2 \mathrm{mM}$ L-Glutamine at $37^{\circ} \mathrm{C}$ with 5\% carbon dioxide. The media was supplemented with dried sodium hyaluranon (Lifecore Biomedical, MN, U.S.A) at $100 \mu \mathrm{g} / \mathrm{ml}$ concentration. All experiments were conducted with cells cultured to $60 \%$ confluence. Cigarette smoke condensate (CSC) was prepared by bubbling $40 \mathrm{ml}$ of plain $\alpha$-MEM with four 3R4F Reference Research Cigarettes (The University of Kentucky, College of Agriculture, Lexington, $\mathrm{KY}$ ) at a negative pressure of $10 \mathrm{~cm} \mathrm{H}_{2} \mathrm{O}, \mathrm{pH}$ adjusted to 7.4 and filtered with 0.22 $\mu \mathrm{m}$. This was used as $100 \%$ and diluted as desired in further experiments. The CSC was prepared fresh for each experiment and added to fresh media to achieve the desired concentration (final concentration of 5\% FBS for all conditions). Control media was prepared in a similar fashion except room air was bubbled through the media.

\section{Antibodies, Chemicals \& reagents}

The following antibodies were used: Mouse polyclonal anti-CD44 (R\&D systems), mouse monoclonal anti-CD44 (Santa Cruz Biotechnology, CA), rabbit polyclonal anti-GAPDH (Santa Cruz Biotechnology, CA), mouse monoclonal anti CTTN (Upstate cell signaling solution), rabbit monoclonal anti BIRC5 (Santa Cruz Biotechnology), goat anti-mouse IgG HRP CD44 (Santa Cruz Biotechnology), goat anti-rabbit IgG HRP CD44 (Santa Cruz Biotechnology) antibodies.

\section{Proliferation Assay}

The AlamarBlue ${ }^{\circledR}$ cell proliferation assay (Invitrogen, Carlsbad, CA) was utilized to determine the toxicity of CSC on MSC. MSC were seeded at $2 \times 10^{4}$ cells on Costar black tissue culture plates and 
allowed to attach overnight. The cells were then incubated in media containing various doses of CSC and AlamarBlue ${ }^{\circledR}$ was added to all the wells. Cells were observed at serial time points for morphological changes and fluorometric readings were taken at excitation of $540 \mathrm{~nm}$ and emission $610 \mathrm{~nm}$.

\section{Cell Migration and Invasion Assays}

Migration and invasion chambers were prepared by coating cell culture inserts $(12 \mu \mathrm{m}$ pore size, Millipore, MA) with $200 \mu \mathrm{g} / \mathrm{ml}$ of Matrigel ${ }^{\mathrm{TM}}$ (BD Biosciences, MA), which serves as a basement membrane substitute corresponding to the basement membrane of blood vessels. Assays were conducted using MSC exposed to CSE, control media as well as with media containing RNAi of CD44 for four hours as previously described [40]. After trypsinization cells were resuspended in $\alpha$-MEM with $1 \%$ FBS at $5 \times 10^{5}$ cells/200 $\mu \mathrm{l} /$ well, added to the upper chamber and then incubated for $24-\mathrm{hrs}$ at $37^{\circ} \mathrm{C}$. After 4 hours, the cells were detached and seeded into transwell

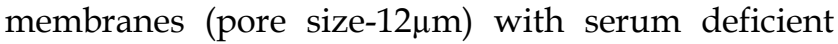
media placed in 12 well plates containing 1\% FBS containing MEM -alpha medium. The 12 well plates were incubated for 24 hours at $5 \% \mathrm{CO}_{2}$ and $37^{\circ} \mathrm{C}$. At the end of 24 hours, the upper surface of the filter was then wiped with a cotton swab to remove non-migratory cells. The cells attached to the other side of filter were stained with a Diff-Quick stain set (Dade Behring Inc.), photographed under a phase-contrast microscope and the total number of stained cells for each filter was tabulated using a counting grid.

\section{Western blotting}

Cell lysates were prepared from cells treated for 24 hours with bubbled CSC or control media and the final protein concentration in the supernatant was determined using the Bradford Protein Assay Reagent (Sigma-Aldrich, St. Louis, MO) as previously described [40]. Samples $(60 \mu \mathrm{g})$ were boiled for 5 minutes in an equal volume of reducing buffer and resolved on $12 \%$ polyacrylamide gels and electroblotted onto PVDF membranes. Membranes were probed with mouse anti-CD44 (1:2000 dilution, R\& D systems), mouse monoclonal anti CTTN antibody (1:2000 dilution, Upstate cell signaling solution), rabbit monoclonal anti BIRC5 antibody (1:2000 dilution, Santa Cruz Biotechnology)and goat anti-GAPDH antibody (1:2000 dilution, Santa Cruz, CA) and detected with a goat anti-mouse and donkey anti-goat IgG-HRP (1:5000 dilution, Santa Cruz, CA) using chemiluminescence (Supersignal West Femto, Pierce).

\section{Preparation of RNA samples}

Cells were treated with CSC and harvested after 12 and 24 hours. RNA was isolated using RNeasy Mini kit (Qiagen) according to the manufacturer's recommendations. Isolated RNA samples were assessed for quantity and purity (NanoDrop Technologies Inc. DE, USA) and stored at $-80{ }^{\circ} \mathrm{C}$ for further analysis.

\section{RT-PCR Analysis}

For RT-PCR analysis (Qiagen One Step RT-PCR kit), $1.0 \mu \mathrm{g}$ of total RNA was reverse transcribed using standard reagents according to the manufacturer's instructions. Samples were incubated in the PTC-200 Thermal Cycler for reverse transcription at $50^{\circ} \mathrm{C}$ for 30 min, initial PCR activation step at $95^{\circ} \mathrm{C}$ for $5 \mathrm{~min}$ followed by 32 polymerase chain reaction cycles. Each cycle consisted of at $95^{\circ} \mathrm{C}$ for 30 seconds, $56^{\circ} \mathrm{C}$ for 30 seconds, and $68^{\circ} \mathrm{C}$ for 1 minute. The final completion step was carried out at $68^{\circ} \mathrm{C}$ for 10 minutes. The annealing temperatures for various genes were calculated according to their sequence and were optimized. Accordingly, the temperatures we used were: $55^{\circ} \mathrm{C}$ for $\mathrm{CD} 44$ and $\mathrm{CTTN}, 51^{\circ} \mathrm{C}$ for BIRC5 and $58^{\circ} \mathrm{C}$ for GAPDH.

The following oligonucleotide primers were used: CD44: TTTGCATTGCAGTCAACAGTC (sense) and TTACACCCCAATCTTCATGTCCAC (antisense); CTTN: AAAGCTTCAGCAGGCCAC (sense) and TTTGGTCCTGTTTCAAGTTCC (antisense); BIRC5: AGCCCTTTCTCAAGGACCA (sense) and TC AATCCATGGCAGCCAG (antisense); GAPDH: ACC ACAGTCCATGCCATCAC (sense) and TCCACCAC CCTGTTGCTGTA (antisense). The RT-PCR products were examined by electrophoresis in $2 \%$ agarose gel containing $0.2 \mu \mathrm{g} / \mathrm{ml}$ ethidium bromide.

\section{RNAi-Mediated Depletion of CD44}

Specific human CD44 siRNA oligonucleotides (5'-GGAAAUGGUGCAUUUGGUGtt-3' and 3'-CAC CAAAUGCACCAUUUCCtt-5') and a Silencer ${ }^{\circledR}$ Negative Control \#1 siRNA were synthesized commercially (Ambion, TX). Cells were seeded and grown to $50 \%$ confluency, washed twice in sterile PBS, then incubated with a transfection cocktail comprised of OPTI-MEM, Lipofectamine-2000 (Invitrogen) and the siRNA or scrambled oligonucleotides, at final concentrations of 100 and $200 \mathrm{nM}$ for 24 hours at $37^{\circ} \mathrm{C}$. Next day, cells were treated once more with the siRNA cocktail. Transfected cells were incubated at $37^{\circ} \mathrm{C}$ for an additional 24 hours before harvest and were then used in experiments. Depletion of CD44 expression in cells was confirmed by immunoblotting. 


\section{Statistical Analysis}

Differences between mean and standard deviation were assessed for statistical significance using one-way ANOVA with Newman-Keuls Multiple Comparison Test (GraphPad InStat version 5.00, GraphPad Software, CA). $p$ value less than 0.05 was considered significant.

\section{Acknowledgements}

This study was supported by Qatar University Internal grant QUUG-CASDBES-15/16-22, grants from the National Heart Lung Blood Institute of the National Institutes of Health (HL073770) and by the LSU Health Sciences Center Translational Research Initiative. Some of the materials employed in this work were provided by the Tulane Center for Gene Therapy at Tulane University, New Orleans, LA through a grant from NCRR of the NIH, Grant \# P40RR017447.

\section{Authors Contributions}

AO: Conception and design, financial support, provision of study material, analysis and interpretation, manuscript writing and final approval of manuscript; RT: Carried out the experiments, Provision of study material, collection of data, participated in data analysis and interpretation; RLG: participated in data analysis and interpretation, and manuscript writing; AF: participated in collection of data, data analysis and interpretation, and manuscript writing; HZ: participated in manuscript preparation and critical editing; MR: participated to data interpretation and manuscript writing; WDA: participated in data analysis and interpretation, manuscript writing, and final approval of the manuscript.

\section{Competing Interests}

The authors have declared that no competing interest exists.

\section{References}

1. Pontikoglou C, Deschaseaux F, Sensebé L, Papadaki H. Bone Marrow Mesenchymal Stem Cells: Biological Properties and Their Role in Hematopoiesis and Hematopoietic Stem Cell Transplantation. Stem Cell Rev and Rep. 2011; 7: 569-89.

2. Janoff A, Carp H, Laurent P, Raju L. The role of oxidative processes in emphysema. Am Rev Respir Dis. 1983; 127: S7.

3. Krebsbach PH, Kuznetsov SA, Satomura K, Emmons RVB, Rowe DW, Robey PG. BONE FORMATION IN VIVO: COMPARISON OF OSTEOGENESIS BY TRANSPLANTED MOUSE AND HUMAN MARROW STROMAL FIBROBLASTS. Transplantation. 1997; 63: 1059-69.

4. Pereira RF, Halford KW, O'Hara MD, Leeper DB, Sokolov BP, Pollard $\mathrm{MD}$, et al. Cultured adherent cells from marrow can serve as long-lasting precursor cells for bone, cartilage, and lung in irradiated mice. Proceedings of the National Academy of Sciences. 1995; 92: 4857-61.

5. Ahmed SK, Mohammed SA, Khalaf G, Fikry H. Role of Bone Marrow Mesenchymal Stem Cells in the Treatment of CCL(4) Induced Liver
Fibrosis in Albino Rats: A Histological and Immunohistochemical Study. International Journal of Stem Cells. 2014; 7: 87-97.

6. Sadan O, Melamed E, Offen D. Bone-marrow-derived mesenchymal stem cell therapy for neurodegenerative diseases. Expert Opinion on Biological Therapy. 2009; 9: 1487-97.

7. Wei X, Yang X, Han Z-p, Qu F-f, Shao L, Shi Y-f. Mesenchymal stem cells: a new trend for cell therapy. Acta Pharmacol Sin. 2013; 34: 747-54.

8. Law MR, Hackshaw AK. A meta-analysis of cigarette smoking, bone mineral density and risk of hip fracture: recognition of a major effect. 1997.

9. van Eeden S, Hogg J. The response of human bone marrow to chronic cigarette smoking. European Respiratory Journal. 2000; 15: 915-21.

10. Ponta H, Sherman L, Herrlich PA. CD44: From adhesion molecules to signalling regulators. Nat Rev Mol Cell Biol. 2003; 4: 33-45.

11. Goodison S, Urquidi V, Tarin D. CD44 cell adhesion molecules. Molecular Pathology. 1999; 52: 189-96.

12. Misra S, Heldin P, Hascall VC, Karamanos NK, Skandalis SS, Markwald $\mathrm{RR}$, et al. HA/CD44 interactions as potential targets for cancer therapy. The FEBS journal. 2011; 278: 1429-43.

13. Shteyngart B, Chaiwiriyakul S, Wong J, Cantor JO. Preferential binding of lysozyme to elastic fibres in pulmonary emphysema. Thorax. 1998; 53: 193-6.

14. Udabage L, Brownlee GR, Nilsson SK, Brown TJ. The over-expression of HAS2, Hyal-2 and CD44 is implicated in the invasiveness of breast cancer. Experimental Cell Research. 2005; 310: 205-17.

15. Hill A, McFarlane S, Mulligan K, Gillespie H, Draffin JE, Trimble A, et al. Cortactin underpins CD44-promoted invasion and adhesion of breast cancer cells to bone marrow endothelial cells. Oncogene. 2006; 25: 6079-91.

16. Ouhtit A, Matrougui K, Bengrine A, Koochekpour S, Zerfaoui M, Yousief Z. Survivin is not only a death encounter but also a survival protein for invading tumor cells. Front Biosci. 2007 12: 10.

17. Ouhtit A, Madani S, Gupta I, Shanmuganathan S, Abdraboh ME, Al-Riyami H, Al-Farsi YM, Raj MH. TGF- $\beta 2$ : A Novel Target of CD44-Promoted Breast Cancer Invasion. J Cancer 2013; 4: 7.

18. Khaldoyanidi S, Sikora L, Orlovskaya I, Matrosova V, Kozlov V, Sriramarao P. Correlation between nicotine-induced inhibition of hematopoiesis and decreased CD44 expression on bone marrow stromal cells. 2001

19. Liu X, Kohyama T, Kobayashi T, Abe S, Kim H, Reed E, et al. Cigarette smoke extract inhibits chemotaxis and collagen gel contraction mediated by human bone marrow osteoprogenitor cells and osteoblast-like cells. Osteoporos Int. 2003; 14: 235-42.

20. Journal of Basic and Clinical Physiology and Pharmacology. 1986.

21. Tipton DA, Dabbous MK. Effects of Nicotine on Proliferation and Extracellular Matrix Production of Human Gingival Fibroblasts In Vitro. Journal of Periodontology. 1995; 66: 1056-64.

22. Naor D, Nedvetzki S, Golan I, Melnik L, Faitelson Y. CD44 in Cancer. Critical Reviews in Clinical Laboratory Sciences. 2002; 39: 527-79.

23. Aruffo A, Stamenkovic I, Melnick M, Underhill CB, Seed B. CD44 is the principal cell surface receptor for hyaluronate. Cell. 1990; 61: 1303-13.

24. Ishii S, Ford R, Thomas P, Nachman A, Steele Jr G, Jessup JM. CD44 participates in the adhesion of human colorectal carcinoma cells to laminin and type IV collagen. Surgical Oncology. 1993; 2: 255-64.

25. Jalkanen S, Jalkanen M. Lymphocyte CD44 binds the COOH-terminal heparin-binding domain of fibronectin. The Journal of Cell Biology. 1992; 116: 817-25

26. Weber GF, Ashkar S, Glimcher MJ, Cantor H. Receptor-Ligand Interaction Between CD44 and Osteopontin (Eta-1). Science. 1996; 271: 509-12.

27. Lokeshwar VB, Bourguignon LY. Post-translational protein modification and expression of ankyrin-binding site(s) in GP85 (Pgp-1/CD44) and its biosynthetic precursors during T-lymphoma membrane biosynthesis. Journal of Biological Chemistry. 1991; 266: 17983-9.

28. Kemp K, Morse R, Sanders K, Hows J, Donaldson C. Alkylating chemotherapeutic agents cyclophosphamide and melphalan cause functional injury to human bone marrow-derived mesenchymal stem cells. Ann Hematol. 2011; 90: 777-89.

29. Pandit TS, Sikora L, Muralidhar G, Rao SP, Sriramarao P. Sustained Exposure to Nicotine Leads to Extramedullary Hematopoiesis in the Spleen. STEM CELLS. 2006; 24: 2373-81.

30. Ghallab N, Shaker O. Salivary-Soluble CD44 Levels in Smokers and Non-Smokers With Chronic Periodontitis: A Pilot Study. Journal of Periodontology. 2010; 81: 710-7.

31. Scott DA, Stapleton JA, Palmer RM, Wilson RF, Sutherland G, Coward PY, et al. Plasma Concentrations of Reputed Tumor-associated Soluble CD44 Isoforms (v5 and v6) in Smokers Are Dose Related and Decline on Smoking Cessation. Cancer Epidemiology Biomarkers \& Prevention. 2000; 9: 1211-4. 
32. Palmer RM, Stapleton JA, Sutherland G, Coward PY, Wilson RF, Scott DA. Effect of nicotine replacement and quitting smoking on circulating adhesion molecule profiles (sICAM-1, sCD44v5, sCD44v6). European Journal of Clinical Investigation. 2002; 32: 852-7.

33. Zhu H, Mitsuhashi N, Klein A, Barsky LW, Weinberg K, Barr ML, et al. The Role of the Hyaluronan Receptor CD44 in Mesenchymal Stem Cell Migration in the Extracellular Matrix. STEM CELLS. 2006; 24: 928-35.

34. Sackstein R, Merzaban JS, Cain DW, Dagia NM, Spencer JA, Lin CP, et al. Ex vivo glycan engineering of CD44 programs human multipotent mesenchymal stromal cell trafficking to bone. Nat Med. 2008; 14: 181-7.

35. Konno K, Arai H, Motomiya M, Nagai H, Ito $M$, Sato $H$, et al. A biochemical study on glycosaminoglycans (mucopolysaccharides) in emphysematous and in aged lungs. The American review of respiratory disease. 1982; 126: 797-801.

36. Petrey AC, de la Motte CA. Hyaluronan, a Crucial Regulator of Inflammation. Frontiers in Immunology. 2014; 5: 101.

37. Abdraboh ME, Gaur RL, Hollenbach AD, Sandquist D, Raj MHG, Ouhtit A. Survivin Is a Novel Target of CD44-Promoted Breast Tumor Invasion. The American Journal of Pathology. 2011; 179: 555-63.

38. Fangusaro JR, Jiang Y, Holloway MP, Caldas H, Singh V, Boue DR, et al. Survivin, Survivin-2B, and Survivin-deItaEx3 expression in medulloblastoma: biologic markers of tumour morphology and clinical outcome. Br J Cancer. 2005; 92: 359-65.

39. Vermeulen M, Le Pesteur F, Gagnerault M-C, Mary J-Y, Sainteny F, Lepault F. Role of Adhesion Molecules in the Homing and Mobilization of Murine Hematopoietic Stem and Progenitor Cells. 1998.

40. Draffin JE, McFarlane S, Hill A, Johnston PG, Waugh DJJ. CD44 Potentiates the Adherence of Metastatic Prostate and Breast Cancer Cells to Bone Marrow Endothelial Cells. Cancer Research. 2004; 64: 5702-11. 